\title{
Gravity Effects in Inclined Air Showers Induced by Cosmic Neutrinos
}

\author{
A.V. Kisselev* \\ Institute for High Energy Physics, 142281 Protvino, Russia
}

\begin{abstract}
The Randall-Sundrum model with a small curvature is considered in which five-dimensional Planck scale lies in the TeV region. The cross sections for interactions of ultra-high energy cosmic neutrinos with nucleons are calculated. It is shown that effects related with Kaluza-Klein graviton excitations can be detected in deeply penetrating inclined air showers induced by these neutrinos. The expected number of the inclined air showers at the Auger Observatory is estimated as a function of two parameters of the model.
\end{abstract}

\section{WARPED EXTRA DIMENSION WITH THE SMALL CURVATURE}

One of the most important problems of the modern particle physics is the hierarchy problem, i.e. unnaturally large ratio of the gravity scale $\left(10^{19} \mathrm{GeV}\right)$ to the electroweak scale $\left(10^{2} \mathrm{GeV}\right)$. To solve this problem, a number of theories with large spacial extra dimensions have been proposed [1]. However, they could only explain the huge value of the Planck mass by introducing another large scale, namely, the size of extra flat dimensions. Thus, the hierarchy problem was not really solved, but reformulated in terms of this new scale.

The model which does solve the problem most economically is the Randall-Sundrum (RS) model [2] with a single extra dimension and warped background metric [3]:

$d s^{2}=e^{2 \kappa(\pi r c-|y|)} \eta_{\mu \nu} d x^{\mu} d x^{\nu}+d y^{2}$.

Here $y=r_{c} \theta(-\pi \leq \theta \leq \pi), r_{c}$ is the "radius" of the extra dimension, while $\left\{x^{\mu}\right\}, \mu=0,1,2,3$, are the coordinates in four-dimensional space-time. The parameter $\kappa$ defines the scalar curvature in five dimensions. Note that the points $\left(x^{\mu}\right.$, $y)$ and $\left(x^{u},-y\right)$ are identified, and the periodicity condition, $\left(x^{\mu}, y\right)=\left(x_{\mu}, y+2 \pi r_{c}\right)$, is imposed. The tensor $\eta_{\mu v}$ is the Minkowski metric.

It is assumed that there are two 3-dimensional branes with equal and opposite tensions located at the points $y=0$ (called the Plank brane) and $y=\pi r_{c}$ (referred to as the TeV brane). All SM fields are confined to the TeV brane, while the gravity propagates in five dimensions. The following relation between the 4-dimensional (reduced) Planck mass, $\bar{M}_{\mathrm{P} 1}$, and (reduced) gravity scale in five dimensions, $\bar{M}_{5}$, can be derived:

$\bar{M}_{\mathrm{P} 1}^{2}=\frac{\bar{M}_{5}^{3}}{k}\left(e^{2 \pi k r_{c}}-1\right)$.

The masses of the Kaluza-Klein (KK) graviton excitations are proportional to the curvature parameter $\kappa$ :

$m_{n}=x_{n} \kappa, \mathrm{n}=1,2 \ldots$,

*Address for correspondence to this author at the Institute for High Energy Physics, 142281 Protvino, Russia; E-mail: alexandre.kisselev@ihep.ru where $x_{n}$ are zeros of the Bessel function $J_{1}(x)$. On the $\mathrm{TeV}$ brane, the zero graviton mode, $h_{\mu \nu}^{(0)}$, and massive graviton modes, $h_{\mu \nu}^{(n)}$, are coupled to the energy-momentum tensor of the matter, $T^{\mu \nu}$, as follows:

$$
\mathcal{L}_{\text {int }}=-\frac{1}{\bar{M}_{P l}} T^{\mu v} h_{\mu \nu}^{(0)}-\frac{1}{\Lambda_{\pi}} T^{\mu \nu} \sum_{n=1}^{\infty} h_{\mu v}^{(n)}
$$

with

$\Lambda_{\pi}=\left(\frac{\bar{M}_{5}^{3}}{k}\right)^{1 / 2}$

being the physical scale on this brane. Let us note that the metric (1) differs from that presented in the original paper [2] in which both $\bar{M}_{5}$ and $\kappa$ have to be taken as large as the Planck mass $\left(\bar{M}_{5} \sim \kappa \sim \bar{M}_{\mathrm{P} 1}\right)$. Moreover, the size of the warped extra dimension should be extremely small $\left(r_{c} \simeq\right.$ $60 l_{\mathrm{Pl}}$ ) [3]. Thus, in order to explain the huge value of $\bar{M}_{\mathrm{P} 1}$ in such a scheme, one has to introduce new mass scales of the same order, namely, $\bar{M}_{5}$, $\kappa$, and $r_{c}^{-1}$.

However, the hierarchy problem can be successfully solved in the RS scenario, but with the metric (1). The equation (2) allows us to consider the small curvature option of the RS model [3-5]: ${ }^{1}$

$\kappa \sim 1 \mathrm{GeV}, \bar{M}_{5} \sim 1 \mathrm{TeV}$.

In such a case, we get an almost continuous spectrum of lowmass graviton excitations with the lightest mass equal to $3.83 \kappa$, and small mass splitting $\Delta m \simeq \pi \kappa$. Note that in the standard scenario of the RS model [2] one has a series of KK graviton resonances with the lightest one having the mass around $1 \mathrm{TeV}$.

The RS model with the large extra dimension has been checked by the DELPHI Collaboration [6]. The gravity

${ }^{1}$ For numerical estimates, the region $0.5 \mathrm{GeV}<\kappa<1.5 \mathrm{GeV}$ will be used (see Fig. 3). 
effects were searched for by studying photon energy spectrum in the process $e^{+} e^{-} \rightarrow \gamma+Z_{\perp}$. The limit on $M_{5}$ obtained [6] is:

$$
\bar{M}_{5}>0.92 \mathrm{TeV} \text {. }
$$

The search for the large extra dimensions in the diphoton channel using data collected by the CDF and $\mathrm{D} \varnothing$ Collaborations at $\sqrt{s}=1.96 \mathrm{TeV}$ is presented in Refs. [7]. The measured $p_{\perp}$-distributions are in a good agreement with the SM background, that allowed us to obtain the bound [8]:

$$
\bar{M}_{5}>0.81 \mathrm{TeV} \text {. }
$$

The discovery limit of the LHC in the two-photon production (requiring a $5 \sigma$ effect) has also been derived for two values of the integrated luminosity $\mathcal{L}[8]$ :

$$
\bar{M}_{5}=\left\{\begin{array}{rr}
6.3 \mathrm{TeV}, & \mathcal{L}=100 \mathrm{fb}^{-1} \\
5.1 \mathrm{TeV}, & \mathcal{L}=30 \mathrm{fb}^{-1}
\end{array}\right.
$$

Previously, the gravity effects in the RS model with the small curvature were already looked for in a number of different processes (see Refs. [8-10]). In the present paper we will estimate the upper bound on $\bar{M}_{5}$ which can be reached by the Auger ground array in detecting quasihorizontal air showers induced by ultra-high energy (UHE) cosmic neutrinos.

\section{GRAVITY EFFECTS IN INTERACTIONS OF COSMIC NEUTRINOS WITH ATMOSPHERIC NUCLEONS}

A promising possibility to detect effects induced by lowmass KK gravitons (3) is to look for their contributions to the scattering of the SM fields in the trans-Planckian kinematical region:

$\sqrt{s} \gtrsim \bar{M}_{5} \gg-t$,

with $\sqrt{s}$ being the colliding energy and $t=q_{\perp}^{2}$ four-dimensional momentum transfer. It is also assumed that inequality $\kappa$ « $\bar{M}_{5}$ (6) is satisfied. As we will see below, in the transPlanckian region the gravity contribution to the scattering of UHE cosmic neutrinos off the atmospheric nucleons can dominate the SM contribution.

In the eikonal approximation which is valid in the kinematical region (10), elastic scattering amplitude is given by the sum of gravi-Reggeons, i.e. reggeized gravitons in the $t$-channel. Because of the presence of extra dimension, the Regge trajectory of the graviton is splitting into an infinite sequence of trajectories enumerated by the KK number $n$ [11]:

$\alpha_{n}(t)=2+\alpha_{g}^{\prime} t-\alpha_{g}^{\prime} m_{n}^{2}, \quad n=0,1, \ldots$

In string theories, the slope of the gravi-Reggeons is universal, and $\alpha_{g}^{\prime}=M_{s}^{-2}$, where $M_{s}$ is the string scale. For more details, see Refs. [11].
Correspondingly [3], the gravity Born amplitude for the neutrino scattering off $a$ point-like particle looks like ${ }^{2}$ :

$A_{\text {grav }}^{\mathrm{B}}(s, t)=\frac{\pi \alpha_{g}^{\prime} s^{2}}{2 \Lambda_{\pi}^{2}} \sum_{n \neq 0}\left[i-\cot \frac{\pi \alpha_{n}(t)}{2}\right]\left(\frac{s}{\bar{M}_{5}}\right)^{\alpha_{n}(t)-2}$.

The differential neutrino-proton cross section is of the form:

$\frac{d \sigma}{d y}=\frac{1}{16 \pi s}\left|A_{v \mathrm{p}}(s, t)\right|^{2}$.

The inelasticity $y=-t / s$ defines the fraction of the neutrino energy transferred to the nucleon. $A_{\nu \mathrm{p}}$ is the neutrino-proton amplitude which is related to the eikonal:

$A_{v p}(s, t)=4 \pi i s \int_{0}^{\infty} d b b J_{0}\left(b q_{\perp}\right)\{1-\exp [i \chi(s, b)]\}$.

In its turn, the eikonal is given by the equation:

$\chi(s, b)=\frac{1}{4 \pi s} \int_{0}^{\infty} d q_{\perp} q_{\perp} J_{0}\left(q_{\perp} b\right) A_{v p}^{\mathrm{B}}(s, t)$.

The calculations show that the imaginary part of the eikonal is negligible with respect to its real part, since $\operatorname{Im} \chi / \operatorname{Re} \chi=$ $\mathrm{O}\left(\kappa / \bar{M}_{5}\right)$. That is why we can omit the contribution from inelastic interactions.

The hadronic Born amplitude in (15) is defined by the gravity amplitude (12) and skewed ( $t$-dependent) parton distributions $F_{i}(x, t):^{3}$

$$
A_{v p}^{\mathrm{B}}(s, t)=\sum_{i=q, \bar{q}, g} \int_{0}^{1} d x A_{\text {grav }}^{\mathrm{B}}(x s, t) F_{i}(x, t) .
$$

The $t$-dependent distributions have the Regge-like form [3]:

$F_{i}(x, t)=f_{i}(x) \exp \left[t\left(r_{0}^{2}-\alpha_{P}^{\prime} \ln x\right)\right]$,

where $\alpha_{0}^{\prime}$ is the Pomeron slope, while $f_{i}(x)$ is the distribution of the parton of the type $i$ inside the proton. The values of the parameters are [12]:

$$
r_{0}^{2}=0.62 \mathrm{GeV}^{-2}, \alpha_{P}^{\prime}=0.094 \mathrm{GeV}^{-2} \text {. }
$$

We will use the set of parton distribution functions $f_{i}(x)$ from Ref. [13].

In Fig. (1) and Fig. (2) we present total neutrino-nucleon cross sections calculated by using Eqs. (12)-(16) for two values of the curvature $\kappa$ and different values of the reduced fundamental gravity scale $\bar{M}_{5}$. For comparison, the SM prediction for the neutrino total cross section is presented in both figures which was calculated by using the following expression from Ref. [14] $]^{4}$.

\footnotetext{
${ }^{2}$ Remember that the KK gravitons interact universally with the SM fields (4).

${ }^{3}$ Since $A^{\mathrm{B}} \sim s^{2}$, the integral converges rapidly at $x=0$.

${ }^{4}$ This expression is valid for $10^{7} \mathrm{GeV} \leq E_{v} \leq 10^{12} \mathrm{GeV}$ within $10 \%$ [14].
} 
$\sigma_{\text {tot }}(v N)=7.84 \cdot 10^{-36} \mathrm{~cm}^{2}\left(\frac{E_{v}}{1 \mathrm{GeV}}\right)^{0.363}$

It varies from $2.72 \cdot 10^{-33} \mathrm{~cm}^{2}$ at $E_{v}=10^{7} \mathrm{GeV}$ up to 1.78 . $10^{-31} \mathrm{~cm}^{2}$ at $E_{v}=10^{12} \mathrm{GeV}$.

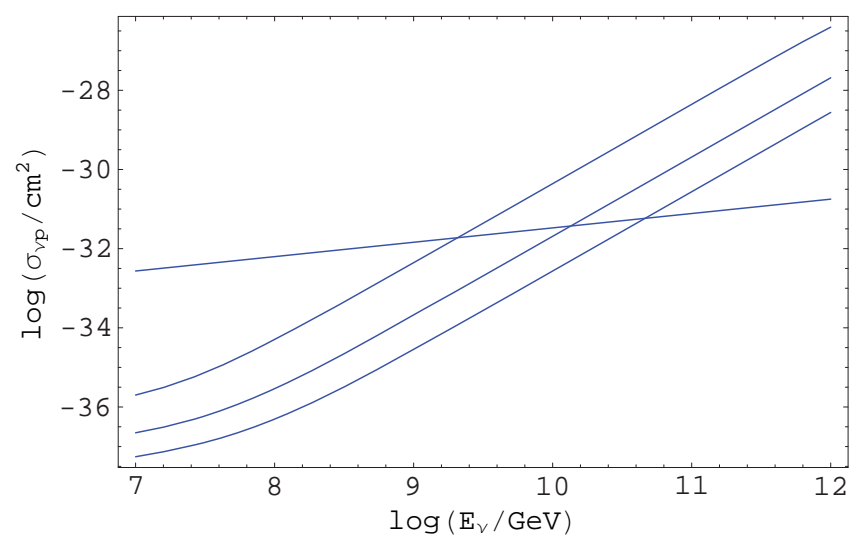

Fig. (1). The total neutrino-proton cross section as a function of the neutrino energy. The curves correspond (from above) to $\bar{M}_{5}=3$, $\mathrm{TeV}, 5 \mathrm{TeV}$, and $7 \mathrm{TeV}$. The parameter $\kappa$ is equal to $100 \mathrm{MeV}$. The straight line: SM total cross section.

Previously, low-scale gravity effects in cosmic neutrino interactions were calculated in models with compactified extra dimensions (see $[15,16]$ and references therein). Recently, the gravity effects on the neutrino-nucleon cross sections in the eikonal approximation were estimated for the case of infinitely thin branes embedded in five extra dimensions [17]. The black hole production cross sections in cosmic neutrino interactions were also calculated (see, for instance, Refs. [18]).

By comparing Figs. (1, 2) with figures from Refs. [17], one can see that the neutrino cross sections in the small curvature scenario of the RS model and those in the ADD model have different energy dependence. The formers are significantly smaller at $E_{v} \lesssim 10^{9} \mathrm{GeV}$, but exceed the ADD cross sections at $E_{v} \gtrsim 10^{10} \mathrm{GeV}$ (at comparable values of gravity scale $\bar{M}_{5}$ in both models).

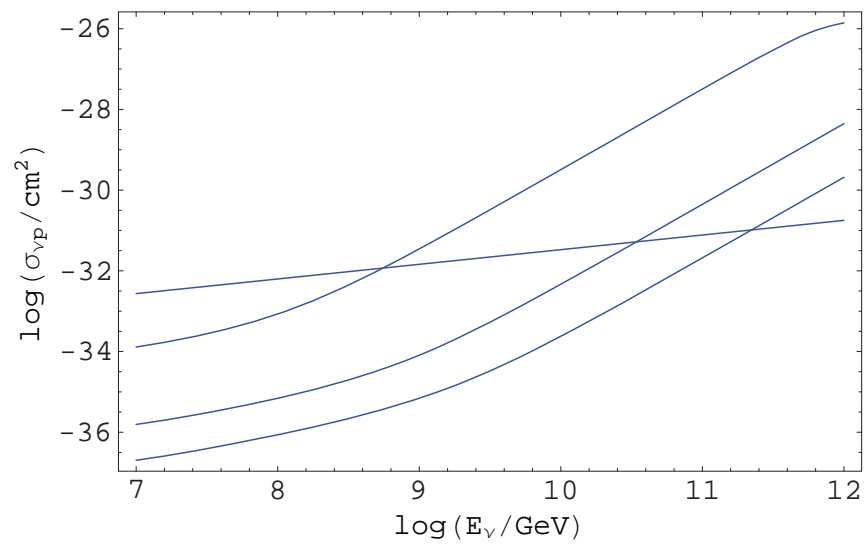

Fig. (2). The total neutrino-proton cross section as a function of the neutrino energy. The curves correspond (from above) to $\bar{M}_{5}=1$, $\mathrm{TeV}, 3 \mathrm{TeV}$, and $5 \mathrm{TeV}$. The parameter $\kappa$ is equal to $1 \mathrm{GeV}$. The straight line: SM total cross section.
The number of neutrino induced air showers is given by

$$
\begin{aligned}
& \frac{d N_{\mathrm{e} v}}{d t}=\int_{E_{\mathrm{th}}}^{E_{\max }} d E_{v} \int_{0}^{1} d y \theta\left(E_{\mathrm{sh}}-E_{\mathrm{th}}\right) \\
& \frac{d \sigma\left(E_{v}\right)}{d y} \Phi\left(E_{v}\right) A_{\mathrm{eff}}\left(E_{\mathrm{sh}}, E_{v}\right),
\end{aligned}
$$

where $E_{v}$ is the energy of the cosmic neutrino, $\Phi\left(E_{v}\right)$ denotes its flux, and

$E_{\mathrm{sh}}=y E_{v}$

is the air shower energy. The effective aperture for the UHE neutrinos is defined by the neutrino flux attenuation $\operatorname{att}\left(E_{v}\right)$ and detector efficiency $P\left(E_{\mathrm{sh}}\right)$ :

$A_{\text {eff }}\left(E_{\mathrm{sh}}, E_{v}\right)=\operatorname{att}\left(E_{v}\right) P\left(E_{\mathrm{sh}}\right) A_{\mathrm{p}}\left(E_{\mathrm{sh}}\right)$.

The attenuation $\operatorname{att}\left(E_{v}\right)$ depends (besides neutrinonucleon total cross section) on $X_{\mathrm{obs}}$, the depth within which air shower is visible for the ground array detector, and $X_{\text {uno, }}$, the minimum atmospheric depth a neutrino must reach in order to induce an observable shower to these detectors.

In order to isolate neutrino-induced events at the Auger Observatory, deeply penetrating quasi-horizontal air showers should be looked for [19, 20]. We impose the following bounds on the zenith angle of the incoming neutrino: $75^{\circ} \leq$ $\theta_{\text {zenith }} \leq 90^{\circ}$. The functions $P\left(E_{\mathrm{sh}}\right)$ and $A_{\mathrm{p}}\left(E_{\mathrm{sh}}\right)$, as well as values of the parameters $X_{\mathrm{obs}}$ and $X_{\text {uno, }}$ are taken from Ref. [21]. In particular, the deeply penetrating events must satisfy the condition $X_{\text {uno }} \geq 1700 \mathrm{~g} / \mathrm{cm}^{2}$. Since, on average, ultrahigh energy air shower develops to its maximum after traversing $800 \mathrm{~g} / \mathrm{cm}^{2}, 5$ it is set $X_{\mathrm{obs}}=1300 \mathrm{~g} / \mathrm{cm}^{2}$ (see [20, 21] for more details).

The threshold energy in (20) is taken to be $E_{\mathrm{th}}=5 \cdot 10^{7}$ $\mathrm{GeV}$, and the maximum energy $E_{\max }=10^{12} \mathrm{GeV}$. The result of our calculations for the Waxman-Bahcall neutrino flux [22] is presented in Fig. (3). It shows the rate of the inclined air showers at the Auger detector as a function of two parameters of the model.

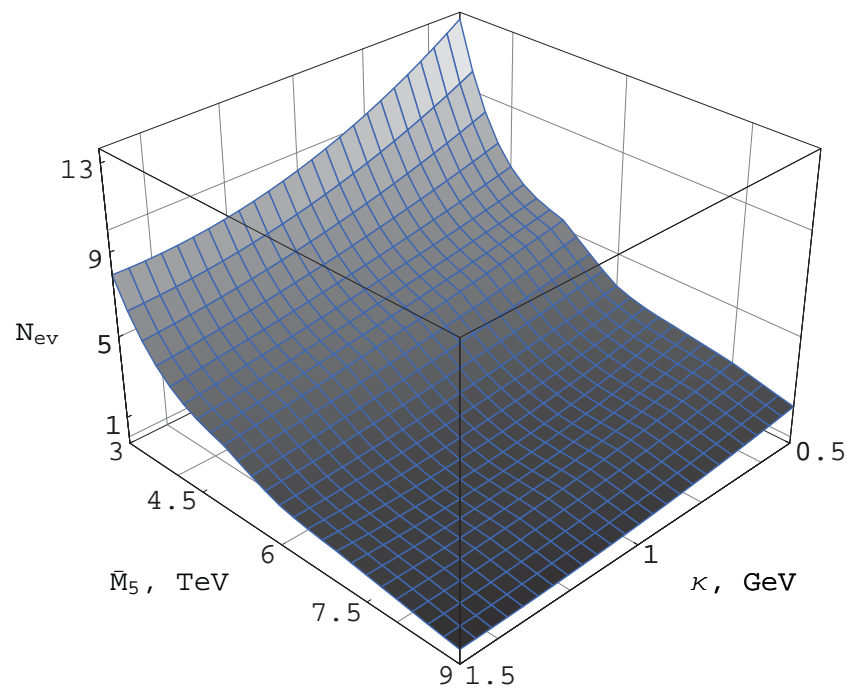

Fig. (3). The expected rate of the neutrino induced inclined air showers $\left(75^{\circ} \leq \theta_{\text {zenith }} \leq 90^{\circ}\right)$ at the Auger Observatory for the Waxman-Bahcall flux (in $\mathrm{yr}^{-1}$ ).

${ }^{5}$ It corresponds to $X \max \geq 2500 \mathrm{~g} / \mathrm{cm}^{2}$, where $X \max$ is the shower maximum. 
The number of the inclined air showers at $\bar{M}_{5}=7 \mathrm{TeV}$, $\bar{M}_{5}=8 \mathrm{TeV}$, and $\bar{M}_{5}=9 \mathrm{TeV}$ are presented in Table $\mathbf{1}$ for three different values of the parameter $\kappa$. Our SM prediction is 0.13 events per year. It can be compared with the estimate from Ref. [15], $0.22 \mathrm{SM}$ events per year for $\theta_{\text {zenith }} \geq 70^{\circ}$.

Table 1. The Number of the Inclined Neutrino Induced Air Showers (in $\mathrm{yr}^{-1}$ ) at Several Values of the Parameters $\bar{M}_{5}$ and $\kappa$

\begin{tabular}{|c|c|c|c|}
\hline & $\overline{\boldsymbol{M}}_{\mathbf{5}}=\mathbf{7} \mathbf{~ T e V}$ & $\overline{\boldsymbol{M}}_{\mathbf{5}}=\mathbf{8} \mathbf{~ T e V}$ & $\overline{\boldsymbol{M}}_{\mathbf{5}}=\mathbf{9} \mathbf{~ T e V}$ \\
\hline \hline$\kappa=0.5 \mathrm{GeV}$ & 2.69 & 2.53 & 1.54 \\
\hline$\kappa=1.0 \mathrm{GeV}$ & 1.32 & 1.17 & 0.68 \\
\hline$\kappa=1.5 \mathrm{GeV}$ & 1.18 & 0.77 & 0.37 \\
\hline
\end{tabular}

Taking into account that systematic errors of SM cross section can be factor 3 , we conclude that the search limit of the Auger Observatory for the 5-dimensional Planck scale $\bar{M}_{5}$ varies from $7 \mathrm{TeV}$ to $9 \mathrm{TeV}$ when $\kappa$ varies from 1.5 $\mathrm{GeV}$ to $0.5 \mathrm{GeV}$. These values are closed to the discovery limit of the LHC which was derived recently in the framework of the same scenario (see Eq. (9)).

Since our scheme has only one extra dimension, the sum over KK excitations is UV-finite, contrary to the ADD scheme with two or more extra dimensions (see, for instance, Sec. II.C of the first reference in [18]). In any case, a possible UV cutoff is irrelevant, since it should be equal to (or larger than) the scale $\bar{M}_{5}$, while the masses of the first KK states are much smaller than $\bar{M}_{5}$.

Thus, our predictions (Figs. 1-3) depend only on the 5dimensional gravity scale $\bar{M}_{5}$ and curvature parameter $\kappa$.

\section{REFERENCES}

[1] Arkani-Hamed N, Dimopoulos S, Dvali G. The hierarchy problem and new dimensions at a millimeter. Phys Lett 1998; B429: 263; Phenomenology, astrophysics, and cosmology of theories with submillimeter dimensions and $\mathrm{TeV}$ scale quantum gravity. Phys Rev 1999; D59: 086004; Antoniadis I, Arkani-Hamed N, Dimopoulos S, Dvali G. New dimensions at a millimeter to a Fermi and superstrings at a TeV. Phys Lett 1998; B436: 257.

[2] Randall L, Sundrum R. A large mass hierarchy from a small extra dimension. Phys Rev Lett 1999; 83: 3370.

[3] Kisselev AV, Petrov VA. Gravi-reggeons and trans-planckian scattering in models with one extra dimension. Phys Rev 2005; D71: 124032 .
[4] Kisselev AV. Virtual gravitons and brane field scattering in the Randall-Sundrum model with a small curvature. Phys Rev 2006; D73: 024007.

[5] Giudice GF, Plehn T, Strumia A. Graviton collider effects in one and more extra dimensions. Nucl Phys 2005; B706: 455.

[6] Ask S, Hedberg V, Navarria FL. Contributed paper for the $23^{\text {rd }}$ International Symposium on Lepton-Photon Interactions at High Energy (LP07); Daegu, Korea 2007.

[7] Abbott B, Abolins M, Abramov V, et al. Search for large extra dimensions in dielectron and diphoton production. Phys Rev Lett 2001; 86: 1156; Acosta D, Adelman J, Affolder T, et al. Measurement of the cross section for prompt diphoton production in $p \bar{p}$

collisions at $\sqrt{s}=1.96 \mathrm{TeV}$. Phys Rev Lett 2005; 95: 022003.

[8] Kisselev AV. RS model with a small curvature and two-photon production at the LHC. J High Energy Phys 2008; 9: 39.

[9] Kisselev AV, Petrov VA, Ryutin RA. 5-dimensional quantum gravity effects in exclusive double diffractive events. Phys Lett 2005; B630: 100.

[10] Kisselev AV. RS model with the small curvature and Bhabha scattering at the ILC. J High Energy Phys 2007; 3: 6.

[11] Kisselev AV, Petrov VA. Gravireggeons in extra dimensions and interaction of ultra-high energy cosmic neutrinos with nucleons. Eur Phys J 2004; C36: 103; Eikonal amplitude in the gravireggeon model at superplanckian energies. Eur Phys J 2004; C37: 241.

[12] Petrov VA, Prokudin AV. The first three pomerons. Eur Phys J 2002; C23: 135.

[13] Alekhin SI. Parton distribution functions from the precise NNLO QCD fit. JETP Lett 2005; 82: 628.

[14] Gandhi R, Quigg C, Reno MH, Sarcevic I. Neutrino interactions at ultrahigh energies. Phys Rev 1998; D58: 093009.

[15] Anchordoqui LA, Han T, Hooper D, Sarkar S. Exotic neutrino interactions at the Pierre Auger Observatory. Astropart Phys 2006; 25: 14 .

[16] Hussain S, McKay DW. Energy and angular distribution of upward UHE neutrinos and signals of low scale gravity: Role of tau decay. Phys Rev 2004; D69: 085004.

[17] Sessolo EM, McKay DW. Eikonal contribution to ultra high energy neutrino-nucleon cross sections in low scale gravity models. Phys Lett 2008; B668: 396.

[18] Anchordoqui LA, Feng JL, Goldberg H, Shapere AD. Black holes from cosmic rays: Probes of extra dimensions and new limits on TeV-scale gravity. Phys Rev 2002; D65: 124027; Feng JL, Shapere AD. Black hole production by cosmic rays. Phys Rev Lett 2002; 88: 021303; Kowalski M, Ringwald A, Tu H. Black holes at neutrino telescopes. Phys Lett 2002; B529: 1; Alvarez-Mũnitz J, Feng JL, Halzen F, Hooper D. Detecting microscopic black holes with neutrino telescopes. Phys Rev 2002; D65: 124015; Anchordoqui LA, Glenz MM, Parker L. Black holes at the IceCube neutrino telescope. Phys Rev 2007; D75: 024011.

[19] Berezinsky VS, Zatsepin GT. Cosmic rays at ultra high energies (neutrino?). Phys Lett 1969; B28: 423; Berezinsky VS, Smirnov AY. Cosmic neutrinos of ultra-high energies and detection possibility. Astrophys Space Sci 1975; 32: 461.

[20] Zas E. Neutrino detection with inclined air showers. N J Phys 2005; 7: 130 .

[21] Anchordoqui LA, Fodor Z, Katz SD, Ringwald A, Tu H. Upper bounds on the neutrino-nucleon inelastic cross section. J Cosmol Astropart Phys 2005; 6: 13.

[22] Bahcall J, Waxman E. High energy astrophysical neutrinos: The upper bound is robust. Phys Rev 2001; D64: 023002.

(C) A.V. Kisselev; Licensee Bentham Open.

This is an open access article licensed under the terms of the Creative Commons Attribution Non-Commercial License (http://creativecommons.org/licenses/bync/3.0/), which permits unrestricted, non-commercial use, distribution and reproduction in any medium, provided the work is properly cited.

${ }^{6}$ Remember that we imposed stronger condition $\theta$ zenith $\geq 75^{\circ}$ during our calculations. 